\title{
Motivação para Aprender na Formação Superior em Saúde
}

\author{
Leandro Dias de Araujo ${ }^{1}$ \\ Márcia Maria Peruzzi Elia da Mota ${ }^{1}$ \\ ${ }^{1}$ Universidade Salgado de Oliveira
}

\begin{abstract}
Resumo
Estudos têm demonstrado que a motivação para aprender está associada ao bom desempenho acadêmico. O presente estudo objetivou investigar se há diferenças na motivação para aprender entre os cursos de Fisioterapia, Educação Física e Enfermagem, e a influência do estágio supervisionado. Participaram deste estudo 500 universitários de ambos os sexos, entre 18 e 58 anos $(m=26,43 ; d p=6,97)$, do primeiro ao décimo período, sendo que $279(55,8 \%)$ já haviam realizado estágio curricular. A coleta de dados foi realizada por meio da Escala de Avaliação da Motivação para Aprender de Alunos Universitários. Os resultados mostraram que alunos dos cursos de Enfermagem e Fisioterapia eram mais motivados que os de Educação Física. Em nenhum dos cursos foram encontradas diferenças de motivação entre sexo masculino e feminino. A análise que comparou os alunos que fizeram estágio e não fizeram estágio também não foi significativa.

Palavras-chave: motivação, estágio curricular supervisionado, motivação acadêmica, motivação e aprendizagem
\end{abstract}

\section{Motivation to Learn in Health College Training}

\begin{abstract}
Studies have shown that motivation to learn is associated to good academic performance. Our goal was to investigate differences in learning motivation among the courses of physiotherapy, physical education, and nursery, and the influence of a supervised internship. Participants included 500 university students of both sexes, aged between 18 and 58 years $(M=26.43$; SD =6.97), from the first to the tenth period, of which $279(55.8 \%)$ had already completed a curricular internship. Data collection was conducted with the Scale for evaluation of motivation lo learn of university students. Results showed that the nursery and physiotherapy students were more motivated than those of physical education courses. No differences in motivation were not found between men and women in any of the courses. The analysis that compared the students who completed an internship and those who did not, showed no relevant significance.

Keywords: motivation; supervised curricular internship; academic motivation; motivation and learning
\end{abstract}

\section{Motivación para Aprender en Educación Superior en Salud}

\begin{abstract}
Resumen
Los estudios han demostrado que la motivación para aprender está asociada a un buen desempeño académico. El objetivo de este estudio fue investigar si hay diferencias de motivación entre los cursos de fisioterapia, educación física y enfermería, y la influencia de las pasantías supervisadas. Participaron en este estudio 500 estudiantes universitarios de ambos sexos, entre 18 y 58 años $(m=26,43 ; d e=6,97)$, del primer al décimo período, y $279(55,8 \%)$ ya habían realizado esas pasantías supervisadas. La recopilación de datos se llevó a cabo a través de la Escala de Evaluación de Motivación para aprender de los Alumnos Universitarios. Los resultados mostraron que los alumnos de los cursos de enfermería y fisioterapia estaban más motivados que los de educación física. En ninguno de los cursos se encontraron diferencias de motivación por causa del sexo. El análisis que comparó a los estudiantes que hicieron pasantías supervisadas y los que no hicieron tampoco fue significativo. Palabras clave: motivación, pasantías curriculares supervisadas, motivación académica, motivación y aprendizaje
\end{abstract}

Nos últimos anos, segundo o Instituto Nacional de Estudos e Pesquisas Educacionais Anísio Teixeira (INEP), houve um aumento do número de estudantes universitários nos cursos superiores. Entre 2006 e 2016, houve um crescimento de $62,8 \%$, com média anual de $5 \%$, e trouxe para essa etapa da escolaridade velhas preocupações dos psicólogos, educadores e daqueles que estudam o ensino de modo geral. A evasão é um desses problemas gerais que, apesar do crescimento no número de concluintes apresentado pelo INEP, ainda tem taxas que ficam acima de $20 \%$, representando um desperdício de recursos investidos nesses estudantes e acabam acarretando em perdas que trazem impactos sociais e econômicos. Além da evasão, a adaptação acadêmica, mudanças de curso, baixas avaliações nos exames nacionais também despertam o interesse dos pesquisadores para os fatores que promovem o bom desempenho acadêmico dos universitários e que podem afetar às práticas profissionais desses estudantes (Soares et al., 2014).

Outro fator importante é a motivação para aprender. Boruchovitch (1994) aponta que o sucesso acadêmico e o profissional não dependem apenas da inteligência já que a motivação para aprender está associada ao bom 
desempenho acadêmico. Motivação é definida etimologicamente como "ação de pôr em movimento" (Bzuneck, 2004 , p. 9) sendo sua origem da palavra latina movere que significa mover, condição do organismo que influencia a direção do comportamento, o impulso interno que leva uma ação (Ferreira, 2008). De acordo com o dicionário Houaiss, motivar é "dar motivo a; causar, provocar; prender atenção de; interessar; apresentar como motivo ou causa de; alegar; ser motivação para; estimular, impulsionar" (Houaiss; Villar; Franco, 2012 p.533). Motivação para aprender, por sua vez, diz respeito às ações direcionadas ao aprendizado.

As teorias da motivação para aprendizagem, em geral, focaram em metas e objetivos a serem alcançados (Deci \& Ryan, 2000). Deci e Ryan (2000a, 2000b) argumentam que a motivação para aprender pode ser estudada a partir de uma abordagem mais ampla. Neste artigo, será utilizada como arcabouço teórico a teoria da autodeterminação, elaborada no ano de 1981 por Richard M. Ryan e Edward L. Deci. Essa Teoria sugere que, para além das metas, devem-se considerar as necessidades dos estudantes. Deci e Ryan (2000) apontam que há necessidades psicológicas e de bem-estar que irão potencializar as metas e que vão influenciar os processos regulatórios usados para se atingi-las. Os autores apontam três tipos de necessidades psicológicas, são elas: a de competência, pertencimento e autonomia. A competência refere-se ao grau de efetividade para se engajar e fazer atividades; o pertencimento é definido como as conexões que o sujeito faz com a comunidade em que convive e; a autonomia é o grau pelo qual o indivíduo percebe a si próprio como responsável pelo seu comportamento (Deci \& Ryan, 2002).

Assim, a base inicial da teoria da autodeterminação (Deci \& Ryan, 1985; 2000; Ryan \& Deci, 2000a; 2000b) é a concepção do ser humano como organismo vivo, orientado para o crescimento, desenvolvimento do self e integração com as estruturas sociais. O comportamento, para ser considerado autodeterminado, necessita, então, ser autônomo, autorregulado, ser expressão de um empoderamento psicológico e resultar em autorrealização (Wehmeyer, 1999).

A teoria da autodeterminação diferencia dois tipos de motivação: a intrínseca e a extrínseca. A motivação intrínseca é definida como o comportamento motivado pela atividade em si, pela simples satisfação ou prazer de realizá-la, e a motivação extrínseca, como a realização de atividades como meio instrumental para alcançar eventos externos desejáveis ou escapar de outros indesejáveis. A teoria da autodeterminação estabelece os níveis motivacionais como um continuum, caracterizado por níveis de autodeterminação. Desse modo, os comportamentos motivacionais podem ser compreendidos entre o nível baixo de autodeterminação (desmotivação) e o nível alto (motivação extrínseca e intrínseca). Esse continuum de autodeterminação (Reeve et al., 2004) apresenta seis estilos de motivação. Inicia-se com desmotivação, caracterizada pela ausência da motivação intrínseca e extrínseca, intenção, autodeterminação, pensamento proativo e aspiração em relação ao comportamento-alvo, seguido pela motivação extrínseca, com diferentes tipos de regulação (integrada, identificada, introjetada e externa) e, finalmente, culmina com a motivação intrínseca, sendo o nível mais autodeterminado e autônomo.

Vários fatores afetam a motivação para aprender. Santos, Mognon, Alcará e Lemos (2011) buscaram possíveis diferenças na motivação para aprender em função do sexo, faixa etária, semestre em curso e área de conhecimento. Participaram da pesquisa 302 alunos, sendo 88 do sexo masculino e 214 do feminino, com média de idade de 24,1 anos. A motivação foi avaliada por meio de duas escalas, a Escala de Motivação para Aprendizagem em Universitários (EMAPRE-U) (Zenorini \& Santos, 2010) e a Escala de Avaliação da Motivação para Aprender de Alunos Universitários (EMAU) (Boruchovitch; Costa \& Neves, 2005). Os resultados mostraram diferenças estatisticamente significativas em relação ao sexo. As mulheres obtiveram médias superiores e diferenciaram-se dos homens.

Em outro estudo de Santos et al., (2011), participaram 239 universitários que foram avaliados com a Escala de Avaliação da Vida Acadêmica (EAVA) e a Escala de Motivação para Aprendizagem (EMAPRE). Foram encontradas diferenças significativas em relação ao sexo e ao curso. As mulheres apresentaram melhor resultado no total da escala. O curso de pedagogia também apresentou melhores valores quando comparados a outros cursos. Não houve diferença com relação à idade dos participantes. As autoras não investigaram as razões para as diferenças entre cursos, mas apontam que fatores, como questões pessoais dos discentes, as demandas do curso, e fatores psicossociais, como adaptação a um novo ambiente acadêmico, são como possíveis razões para diferenças na motivação.

Utilizando como base a teoria da autodeterminação, Almeida (2012) também encontrou diferenças entre cursos. A autora realizou a pesquisa de caráter exploratório com 1.269 alunos de instituições públicas e privadas de diversas regiões do país, que cursavam 
bacharelado em cursos de Administração, Saúde, Educação, entre outros. Utilizou-se, como base a versão adaptada da Escala de Avaliação de Motivação Acadêmica (EMA), desenvolvida inicialmente por Vallerand et al., e validada por Guimarães e Bzuneck (2008). Os resultados indicaram comportamentos com regulação identificada, ou seja, os participantes demonstraram tendência para a adoção de regulação autônoma em seus comportamentos. Os alunos valorizaram cursos de formação que proporcionam crescimento pessoal e contribuição para a qualificação técnica; mulheres e alunos mais velhos apresentaram níveis mais autônomos de motivação. A autora, no entanto, não comparou o nível de motivação por curso estudado.

Na mesma perspectiva, Boruchovitch (2008) realizou a pesquisa sobre a motivação de universitários do curso de formação de professores, utilizando-se o instrumento EMA-U (Boruchovitch \& Neves, 2005). Nesse estudo, constataram-se diferenças significativas entre a média dos participantes nas subescalas Motivação Intrínseca - MI e Motivação Extrínseca - ME, sendo que participantes do sexo feminino apresentaram médias significativamente mais elevadas em ambas subescalas. Verificou-se que as médias na motivação intrínseca e extrínseca são, significativamente, mais elevadas na universidade pública do que na universidade particular. Constatou-se também que tanto as motivações intrínseca como a extrínseca aumentaram no avançar da idade.

Em outra pesquisa utilizando a escala de motivação EMA-U, realizada por Marini (2012), participaram 107 estudantes de Pedagogia. O resultado indicou que a motivação extrínseca e total aumentou proporcionalmente com a idade. Guimarães, Bzuneck e Sanches (2002) e Sobral (2003) demonstraram que estudantes do sexo feminino de modo geral apresentaram maior média de motivação intrínseca e que a motivação extrínseca tem demonstrado força quando associada à intrínseca, promovendo seu reforço.

Embora esses estudos tenham apresentando variações em sexo e idade em relação à motivação para aprender, não foi avaliado o papel de experiências acadêmicas, como estágio supervisionado, e diferenças entre cursos foram, também, pouco exploradas. Uma revisão no SciELO e no PEPSIC, bancos de dados que publicam sobre a realidade nacional, não apresentou nenhum texto sobre o papel do estágio supervisionado na motivação para aprender entre 2006 e 2017 quando utilizado na busca os termos "motivação acadêmica", "motivação e aprendizagem", "motivação para aprender" e "estágio curricular supervisionado".
O estágio curricular é um importante aspecto para formação profissional e acadêmica dos estudantes. As exigências das diretrizes curriculares nacionais (DCN) dos cursos da área de saúde, que normatizam esse estágio, foram pensadas para avançar em uma concepção ampliada de saúde, definidas a partir de competências, habilidades gerais e específicas para o perfil profissional generalista, humanista, crítico e reflexivo, esperando que o formado esteja capacitado a atuar em todos os níveis de atenção à saúde, com base no rigor científico e intelectual, conforme determina a Resolução do CES 4, de 19 de fevereiro de 2002 (Brasil, 2002).

A partir da elaboração das Diretrizes Curriculares Nacionais e da lei n. 11.788/2008, conhecida como "nova lei de estágios", foram criadas diretrizes específicas para as diferentes áreas da saúde, fazendo com que o estágio ganhe destaque no processo de formação na educação superior, tornando-se mais presente e dispondo de maior organização nas matrizes curriculares dos cursos. Essa Lei Federal 11.788 de 2008 estabelece o ato educativo escolar supervisionado visando à preparação para o trabalho dos estudantes que frequentam as instituições de educação superior de forma regular, visando o aprendizado de competências próprias da atividade profissional, contextualizando o currículo e os preparando para a vida cidadã e para o trabalho (Brasil, 2008).

O estágio curricular supervisionado objetiva então estimular a relação ensino-aprendizagem. A formação do educando é complementada com o treinamento prático em situação real que, ao mesmo tempo, é educativa, formativa e presta serviços à comunidade, aperfeiçoando o acadêmico em sua totalidade, respeitando o embasamento ético e disciplinar da profissão, procurando desenvolver indivíduos críticos e agentes de transformação social (Rodrigues \& Leitão, 2000).

No campo de prática do estágio na área de saúde, o acadêmico é inserido no contexto de um sistema de saúde universal, igualitário, integral, de qualidade e fundamentado nos princípios de cidadania, buscando a consolidação do SUS. Diante do estágio, o aluno poderá ver a importância de planejar suas ações, assim como a necessidade de aprender a lidar com a flexibilidade perante as decisões tomadas.

A tendência nas organizações de saúde é a busca por profissionais com o maior número de competências para o desempenho do serviço. $\mathrm{O}$ estudante deverá desenvolver competências e, consequentemente, qualificar-se para o mundo do trabalho precocemente impulsionado, durante o estágio, e não após a sua formação (Ruthes; Feldman \& Cunha, 2010). No presente 
estudo, será investigada se há diferenças na motivação para aprender em três cursos na área de saúde e a influência do estágio supervisionado.

\section{Método}

\section{Participantes}

Participaram deste estudo 500 estudantes universitários de ambos os sexos, sendo a maioria do sexo feminino $(n=311 ; 62,2 \%)$, entre 18 e 58 anos $(m=$ 26,43; $d p=6,97$ ), do primeiro ao décimo período de duas faculdades privadas do estado do Rio de Janeiro. Do total da amostra, 279 (55,8\%) já haviam realizado o estágio e $221(44,2 \%)$ ainda não haviam realizado o estágio curricular. Com relação aos cursos realizados, $186(37,2 \%)$ estudantes são do curso de Fisioterapia, sendo 45 do sexo masculino, com idade média de 25,82 anos $(d p=7,32) ; 141$, do sexo feminino com idade média de 25,26 anos $(d p=5,96)$. Dos $106(21,2 \%)$ participantes do curso de Enfermagem, 13 são do sexo masculino, com idade média de 29,84 anos $(d p=6,96)$ e 93, do sexo feminino com idade média de 29,30 anos $(d p=8,14)$. Do curso de Educação Física participaram $208(41,6 \%)$ estudantes, divididos em 131, do sexo masculino e idade média de 25,73 anos $(d p=6,18)$ e 77 , do sexo feminino e idade média de 26,11 anos ( $d p=7,31)$.

\section{Instrumentos}

\section{Questionário Sociodemográfico}

Elaborado pelos autores, o questionário sociodemográfico é composto de três seções, contendo dados pessoais, acadêmicos e socioeconômicos, num total de 15 questões. Os dados pessoais e socioeconômicos apontam a idade, sexo, estado civil e etnia dos participantes, além da participação na vida econômica de sua família. As informações acadêmicas, entre outras questões, descrevem as características da formação acadêmica, bem como o curso de formação e se o estudante fez ou não estágio curricular, dados que norteiam esta pesquisa.

Escala de Avaliação de Motivação Acadêmica para Aprender entre Universitários (EMA-U)

Elaborada por Neves e Boruchovitch (2007), a escala contém 26 itens, sendo 14 questões relacionadas à motivação intrínseca (ex., Eu procuro saber mais sobre os assuntos que gosto, mesmo sem meus professores pedirem) e 12 que dizem respeito à motivação extrínseca (ex., Eu estudo apenas os conteúdos acadêmicos que irão cair na prova). O questionário é respondido por meio de uma escala Likert, com quatro opções de respostas, que valem 4 pontos para a alternativa "Concordo totalmente", 3 pontos para "Concordo parcialmente", 2 pontos para "Discordo parcialmente" e 1 ponto para "Discordo totalmente" nas questões relacionadas à motivação intrínseca. Essa pontuação é invertida para os itens relativos à motivação extrínseca. A pontuação total (soma MI e ME) varia de 26 a 104 pontos e, quanto maior é a pontuação do estudante na escala total, maior é a sua orientação motivacional intrínseca.

No que diz respeito às propriedades psicométricas da escala, foi verificada correlação moderada e significativa entre os dois fatores, motivação intrínseca e extrínseca $(r=0,47 ; p<0,01)$, assim como índices satisfatórios de consistência interna e precisão. Conclui-se que o instrumento tem boas qualidades psicométricas, medindo a orientação geral do aluno em relação à aprendizagem (Boruchovitch, 2008; Boruchovitch \& Neves, 2007).

\section{Procedimentos}

Após a aprovação do Comitê de Ética e Pesquisa da Universidade dos pesquisadores, conforme parecer $\mathrm{n}^{\mathrm{o}}$ 1.775.551, iniciou-se o procedimento de coleta de dados. A amostra foi de conveniência, sendo os alunos inicialmente convidados a participarem da pesquisa e informados dos seus objetivos. Eles assinaram voluntariamente o termo de consentimento de pesquisa, segundo as Diretrizes e Normas Regulamentadoras de Pesquisas Envolvendo Seres Humanos, conforme Resolução no 466, de 12 de dezembro de 2012, do Conselho Nacional de Saúde, e receberam a cópia desse termo (Brasil, 2012). Seguido à obtenção do consentimento, foi realizada a coleta dos dados coletivamente, em sala de aula, no horário previamente cedido pelo professor na Universidade. Foi aplicado o questionário sociodemográfico e a escala EMA-U pelo próprio pesquisador, de acordo com as instruções que acompanham e padronizam sua aplicação.

\section{Procedimento de Análise de Dados}

Iniciou-se a análise pela avaliação da distribuição dos dados. Os dados foram adequados para realização de análises paramétricas. A assimetria dividida pelo desvio padrão da assimetria foi igual a 2 (Tabachnick \& Fidel, 2007). A média da motivação para aprender obtida na escala EMA-U foi calculada para cada grupo de estudante dividido por sexo, tipo de curso, ter ou não 
feito estágio. A seguir realizou-se uma ANOVA fatorial ( $2 \times 2 \times 3)$ com sexo (feminino $\times$ masculino), prática de estágio (ter feito $\mathrm{x}$ não ter feito estágio), o tipo de curso (Fisioterapia x Enfermagem x Educação Física) e o escore na escala de motivação para aprender como fatores. Análises post hoc de Tukey foram realizadas para fazer a comparação das médias par a par.

\section{Resultados}

A Tabela 1 mostra as médias e os desvios padrão da motivação para aprender para cada curso. A inspeção das médias sugere que as variáveis Sexo e Prática de estágio (ter ou não feito estágio) não parecem influenciar a motivação dos alunos. O curso de Educação Física apresentou as menores médias.

Para verificar se as diferenças observadas entre os cursos quanto à motivação para aprender são estatisticamente significativas, bem como se há diferenças quanto a ter ou não cursado estágio supervisionado, ou entre os sexos, uma ANOVA fatorial foi realizada. Os fatores foram Curso (Enfermagem, Fisioterapia e Educação Física), Prática do estágio (fez ou não fez) e Sexo (feminino e masculino). Para comparação entre os cursos, a análise mostrou um resultado significativo $\left(\mathrm{F}_{(2,498)}\right.$ $=5,37$ e $p=0,005)$, verificou-se também o tamanho do efeito, para essa amostra, Beta $(\eta)=0,02$, indicando um tamanho de efeito fraco, mas significativo.

A análise de variância que comparou os alunos que fizeram estágio e não fizeram estágio também não foi significativa $\left(\mathrm{F}_{(1,499)}=1,61 ; p=0,20\right)$ bem como a diferença entre sexos $\left(F_{(1,499)}=0,06 ; p=0,80\right)$. Nenhuma das interações foi significativa, para Sexo x Prática de estágio $\left(\mathrm{F}_{(1,499)}=0,41 ; p=0,52\right)$, para Sexo x Curso $(\mathrm{F}$ $\left.{ }_{(1,499)}=0,74 ; p=0,92\right)$ e para Curso x Prática de estágio $\left(\mathrm{F}_{(1,499)}=1,06 ; p=0,15\right)$. A interação entre Sexo x Estágio x Curso também não foi significativa $\left(\mathrm{F}_{(2,498)}=1,90\right.$; $p=0,15)$. Análises post hoc Tukey, comparando par a par as médias dos Cursos, mostraram que os alunos dos cursos de Enfermagem e Fisioterapia foram mais motivados que os de Educação Física ( $p=0,043$ e $p<0,001$, respectivamente). Não houve diferença significativa na motivação para aprender entre os cursos de Enfermagem e Fisioterapia $(p=0,36)$.

\section{Discussão}

Este estudo procurou investigar se há diferenças na motivação para aprender de estudantes de diferentes cursos da área de saúde e se a motivação é afetada pela prática de estágio. Para buscar resposta sobre essas diferenças, utilizou-se a Escala de Avaliação de Motivação Acadêmica para Aprender entre Universitários (EMA-U), que foi aplicada em estudantes das áreas de Fisioterapia, Enfermagem e Educação Física, que haviam realizado estágio curricular ou que ainda estavam nas séries iniciais, e não tinham cursado o estágio supervisionado. Também se avaliou diferenças entre os sexos.

Os resultados encontrados mostraram que os alunos dos cursos de Enfermagem e Fisioterapia foram mais motivados para aprender do que os de Educação Física, não havendo diferença estatisticamente significativa entre o curso de Enfermagem e Fisioterapia. Também não houve diferença significativa quando comparado os alunos que fizeram estágio e aos que não fizeram estágio e nem entre os sexos.

Diferenças na motivação para aprender entre os diferentes cursos estudados foram encontradas por Santos et al., (2011) que mostrou melhores resultados para o curso de Pedagogia quando comparado a outros cursos, inclusive Fisioterapia. As autoras não investigaram as razões para as diferenças entre cursos, mas apontam que fatores, como questões pessoais dos discentes, as demandas do curso e fatores psicossociais, como adaptação a um novo ambiente acadêmico, como possíveis razões para diferenças na motivação.

No presente estudo, os alunos de Educação Física obtiveram menores escores na motivação para aprender do que os dos demais cursos. Não estava no escopo do estudo investigar as causas das diferenças entre os cursos, mas algumas hipóteses podem ser levantadas. A primeira é a de que a natureza dos cursos de Fisioterapia e de Enfermagem, na qual os estudantes lidam com as questões de saúde dos pacientes desde cedo, pode ser a razão pela qual os alunos se mostrem mais motivados para aprender. Uma segunda hipótese relacionada a essa é a de que os desafios de lidar com a saúde e doença dos pacientes podem motivar os alunos dos cursos de Enfermagem e Fisioterapia a buscar mais informações do que alunos do curso de Educação Física. Embora o curso de Educação Física esteja incluído no grupo dos cursos da área de saúde, apresenta características diferentes em relação às questões ligadas ao cuidado quando comparado aos dois outros cursos estudados.

Ruiz \& Witter (2005) aponta para a importância da motivação para aprender no ensino superior, que é refletida na escolha do curso, no desejo em se ter um diploma ou ingressar no mercado de trabalho. No entanto, para esse autor, o aluno passar por todo 
Tabela 1.

Média de Motivação e Desvio Padrão por Curso, Sexo e Prática De Estágio

\begin{tabular}{|c|c|c|c|c|c|}
\hline Curso & Sexo & Estágio & Média & Desvio Padrão & $\mathbf{n}$ \\
\hline \multirow{9}{*}{ FISIOTERAPIA } & \multirow{3}{*}{ Masculino } & Sim & 83,40 & 5,77 & 15 \\
\hline & & Não & 82,23 & 7,41 & 30 \\
\hline & & Total & 82,62 & 6,86 & 45 \\
\hline & \multirow{3}{*}{ Feminino } & Sim & 83,17 & 7,35 & 79 \\
\hline & & Não & 83,85 & 9,59 & 62 \\
\hline & & Total & 83,47 & 8,39 & 141 \\
\hline & \multirow{3}{*}{ Total } & Sim & 83,21 & 7,09 & 94 \\
\hline & & Não & 83,32 & 8,93 & 92 \\
\hline & & Total & 83,26 & 8,03 & 186 \\
\hline \multirow{9}{*}{ ENFERMAGEM } & \multirow{3}{*}{ Masculino } & Sim & 79,28 & 6,34 & 7 \\
\hline & & Não & 85,50 & 12,04 & 6 \\
\hline & & Total & 82,15 & 9,53 & 13 \\
\hline & \multirow{3}{*}{ Feminino } & Sim & 83,30 & 8,69 & 39 \\
\hline & & Não & 80,55 & 9,89 & 54 \\
\hline & & Total & 81,70 & 9,46 & 93 \\
\hline & \multirow{3}{*}{ Total } & Sim & 82,69 & 8,44 & 46 \\
\hline & & Não & 81,05 & 10,12 & 60 \\
\hline & & Total & 81,76 & 9,42 & 106 \\
\hline \multirow{9}{*}{ EDUCAÇÃO FÍSICA } & \multirow{3}{*}{ Masculino } & Sim & 78,60 & 9,36 & 84 \\
\hline & & Não & 80,17 & 10,14 & 47 \\
\hline & & Total & 79,16 & 9,64 & 131 \\
\hline & \multirow{3}{*}{ Feminino } & Sim & 77,89 & 10,38 & 55 \\
\hline & & Não & 82,13 & 8,81 & 22 \\
\hline & & Total & 79,10 & 10,08 & 77 \\
\hline & \multirow{3}{*}{ Total } & Sim & 78,32 & 9,75 & 139 \\
\hline & & Não & 80,79 & 9,72 & 69 \\
\hline & & Total & 79,14 & 9,78 & 208 \\
\hline \multirow{9}{*}{ TOTAL } & \multirow{3}{*}{ Masculino } & Sim & 79,33 & 8,88 & 106 \\
\hline & & Não & 81,30 & 9,40 & 83 \\
\hline & & Total & 80,19 & 9,14 & 189 \\
\hline & \multirow{3}{*}{ Feminino } & Sim & 81,52 & 9,01 & 173 \\
\hline & & Não & 82,28 & 9,65 & 138 \\
\hline & & Total & 81,86 & 9,29 & 311 \\
\hline & \multirow{3}{*}{ Total } & Sim & 80,69 & 9,01 & 279 \\
\hline & & Não & 81,91 & 9,54 & 221 \\
\hline & & Total & 81,23 & 9,26 & 500 \\
\hline
\end{tabular}

processo de formação nem sempre é tão estimulante, seja na conclusão de disciplinas, atividades práticas ou estágio obrigatório. Outros autores (Barreto \& Barletta, 2010), em contraste, apontam para a diferente relação experimentada pelo aluno e professor, durante o estágio supervisionado, por essa ser mais próxima e possibilitar o aluno a expor melhor suas vulnerabilidades e potencialidades, podem trazer sentimentos diferentes do que 
se acreditava. O estágio poderia motivar mais o aluno a aprender, já que o colocaria em frente à realidade profissional, experimentando o fazer prático de sua futura profissão.

Os achados desse estudo estão mais em consonância com a visão de Ruiz. Os resultados mostraram que a prática de estágio não afetou a motivação para aprender em nenhum dos cursos estudados. Uma possibilidade para explicar esses resultados é o fato de que a reforma curricular pela qual passou os cursos da área de saúde levou aos estudantes desde cedo a ter contato com a realidade profissional que vão encontrar. Assim, embora não tenham a prática supervisionada, tem alguma vivência profissional. Outra possível explicação, específica aos cursos estudados, é que os alunos fazem estágio curricular em áreas que não são do seu interesse para atender às Diretrizes Curriculares Nacionais, que defendem uma formação generalista, humanista, crítica e reflexiva, com ações em todos os níveis de atenção à saúde.

Outra variável que pode afetar a motivação de universitários é o sexo dos participantes (Boruchovitch, 2008; Guimarães, Bzuneck, \& Sanches 2002; Santos et al., 2011; Sobral, 2003). Não foi o caso nessa amostra de estudantes. O efeito do sexo, bem como todas as interações ligadas a ele, não foi significativo. Nessa amostra, foram avaliados apenas alunos do curso de saúde. É possível que a motivação de homens e mulheres seja diferente em função dos ramos escolhidos dentro da profissão.

Ao avaliar a motivação para aprender, quando influenciada pelo estágio curricular, não se procurou saber se os participantes fizeram estágio somente em áreas de interesse, o que provavelmente afetaria a sua motivação. Para se entender melhor as diferenças encontradas, seria importante verificar essa questão em estudos futuros. Também não se verificou diferenças nas motivações intrínsecas e extrínsecas dos participantes. Não estava no escopo desse estudo avaliar tais diferenças, mas estudos futuros devem investigar possíveis divergências nesses tipos de motivação.

Diferentes autores apontam para um universo de experiências que podem marcar a vida acadêmica dos alunos, como o próprio perfil de cada um, questões pessoais, atitudes diante as exigências acadêmicas e até mesmo o papel do professor nas relações de ensino e aprendizagem, afetando a motivação para aprender e o desempenho (Almeida \& Guimarães, 2009, 2012; Cunha \& Carrilho, 2005; Santos, Polydoro, \& Teixeira, 2010; Zenorini \& Santos, 2010). Estudos futuros deveriam explorar, por meio de pesquisas empíricas, as características específicas de cada curso que motivam a aprendizagem de seus alunos. Esses dados são importantes para que se pense em intervenções que aumentam a motivação dos alunos e, assim, que seja possível, por consequência, melhorar o desempenho acadêmico e a qualificação profissional dos estudantes.

\section{Considerações Finais}

Este estudo teve como objetivo investigar possíveis diferenças na motivação para aprender de estudantes de diferentes cursos da área de saúde e se a motivação é afetada pela prática de estágio. Os resultados indicaram diferenças, os alunos dos cursos de Enfermagem e Fisioterapia demonstraram maior motivação para aprender do que os de Educação Física. Embora não estivesse no escopo do estudo investigar as causas dessas diferenças, algumas hipóteses foram levantas. A expectativa dos alunos e a percepção do curso para crescimento pessoal e profissional podem ser fatores que afetam a motivação para aprender. Outro aspecto que poderia ser observado é a relação entre motivação e engajamento, especialmente no caso de estagiários e alunos que não fizeram estágio. É possível que a prática de estágio afete mais o engajamento do que a motivação, embora sejam construtos que partilham variância. Sugere-se explorar essas relações em estudos futuros, uma vez que a literatura aponta a relevância desses fatores para a boa formação do aluno.

Verificam-se algumas limitações do estudo, como não conhecer as vivências profissionais oferecidas e se os estágios obrigatórios realizados foram em áreas de interesse dos alunos. Esses fatores podem afetar a motivação dos estudantes e apresentam novas variáveis possíveis de serem investigadas para se conhecer melhor a relação entre estágio supervisionado e motivação para aprender. O instrumento utilizado, também, não permite determinar se os níveis de motivação encontrados foram altos ou baixos para cada um dos cursos estudados, uma vez que não há ponto de corte ou classificação de níveis motivacionais. Apesar de não oferecer essa informação, foi possível apontar para diferenças na motivação para aprender dos alunos dos cursos estudados.

Tendo em vista a importância da motivação para aprendizagem, esse tema deve ser mais bem explorado. Foram apontados alguns caminhos, como avaliar a motivação intrínseca e extrínseca e sua relação com a prática de estágio, além de identificar variáveis que 
afetam a motivação dos estudantes. Estudos de intervenção também devem ser realizados controlando-se as variáveis que afetam a motivação dos alunos, de modo que se possa melhorar o desempenho acadêmico dos universitários brasileiros. Em conclusão, o estudo aponta que diferentes cursos despertam diferentes níveis de motivação para aprender, mostrando também a importância de se investigar com mais profundidade esse construto para se ter um panorama mais amplo dos fatores que afetam a formação dos universitários da área de saúde.

\section{Referências}

Almeida, A. L. D. J., \& Guimarães, R. B. (2009). O lugar social do fisioterapeuta brasileiro. Fisioterapia e Pesquisa, 16(1), 82-88. doi:10.1590/ S1809-29502009000100015

Almeida, D. M. D. S. (2012). A motivação do aluno no ensino superior: Um estudo exploratório (Dissertação de mestrado). Universidade Estadual de Londrina. Recuperado de http://www.uel.br/ pos/mestredu/images/stories/downloads / dissertacoes/2012/2012_-_ALMEIDA_Debora_ Menegazzo_Sousa.pdf

Barreto, M. C., \& Barletta, J. B. (2010). A supervisão de estágio em psicologia clínica sob as óticas do supervisor e do supervisionando. Cadernos de Gradnação-Ciências Biológicas e da Saúde, 12(12), 155-171. Recuperadodehttps://www.researchgate.net/profile/ Janaina_Barletta2/publication/301675000_A_SUPERVISAO_DE_ESTAGIO_EM_PSICOLOGIA_CLINICA_SOB_AS_OTICAS_DO_ SUPERVISOR_E_DO_SUPERVISIONANDO/ links/5720e00108aed056fa292a8a/A-SUPERVISAO-DE-ESTAGIO-EM-PSICOLOGIA-CLINICASOB-AS-OTICAS-DO-SUPERVISOR-E-DO-SUPERVISIONANDO.pdf

Brasil. Ministério da Educação. Resolução No CNE/ CES 4, de 19 de fevereiro de 2002. Diretrizes Curriculares Nacionais do Curso de Graduação em Fisioterapia.

Brasil. Lei no 11.788 de 25 de setembro de 2008. Dispõe sobre a regulamentação dos estágios. Brasília, DF, 2008

Brasil. Ministério da Saúde. Conselho Nacional de Saúde. Resolução no 466, de 12 de dezembro de 2012. Aprova as diretrizes e normas regulamentadoras de pesquisas envolvendo seres humanos. Brasília: CNS, 2012.

Boruchovitch, E. (1994). As variáveis psicológicas e o processo de aprendizagem: Uma contribuição. Psicologia: Teoria e Pesquisa, 10(1), 129-139. Recuperado de https://s3.amazonaws. com/academia.edu.documents/38515882/ASVARIAVEIS_PSICOLOGICAS_E_O_PROCESSO_DE_APRENDIZAGEM.PDF?AWSAccess KeyId=AKIAIWOWYYGZ2Y53UL3A\&Expir es $=1526251839 \&$ Signature $=$ Kro2FkW8XnzJx7Q q08VT6EerGzc\%3D\&response-content-dispositi on $=$ inline $\% 3 \mathrm{~B} \% 20$ filename $\% 3$ DASVARIAVEIS_ PSICOLOGICAS_E_O_PROCESSO_DE.pdf

Boruchovitch, E. (2008). Escala de motivação para aprender de universitários (EMA-U): propriedades psicométricas. Avaliação Psicológica, 7(2), 127-134. Recuperado de http://www.redalyc.org/articulo. oa?id $=335027184003$

Boruchovitch, E; Costa, E. R., \& Neves, E. R. C. (2005). Estratégias de aprendizagem: contribuições para a formação de professores nos cursos superiores. In: M.C. Joly; A. A. A. Santos, \& F.F. Sisto (Orgs.), Questões do Cotidiano Universitário (pp.239-260). São Paulo: Casa do Psicólogo.

Bzuneck, J. A. (2004). A motivação do aluno: Orientado a metas de realização. Em E. Boruchovitch \& J. A. Bzuneck (Orgs.), A motivação do aluno: Contribuições da Psicologia Contemporânea. (pp.58-77). Petrópolis, RJ: Editora Vozes.

Cunha, S. M., \& Carrilho, D. M. (2005). O processo deadaptação ao ensino superior e o rendimento acadêmico. PsicologiaEscolareEducacional,9(2),215-224.Recuperado de http://pepsic.bvsalud.org/scielo.php?script $=$ sci_ arttext\&pid $=$ S1413-85572005000200004\&lng $=$ pt\& $\mathrm{nrm}=$ iso

Deci, E. L., \& Ryan, R. M. (1985). The general causality orientations scale: Self-determination in personality. Journal of Research in Personality, 19(2), 109-134. doi: 10.1016/0092-6566(85)90023-6

Deci, E. L., \& Ryan, R. M. (2000). The" what" and" why" of goal pursuits: Human needs and the self-determination of behavior. Psychological Inquiry, 11(4), 227-268. doi: 10.1207/S15327965PLI1104_01

Guimarães, S. É. R., Bzuneck, J. A., \& Sanches, S. F. (2002). Psicologia educacional nos cursos de licenciatura: A motivação dos estudantes. Psicologia Escolar 
e Educacional, 6(1), 11-19. Recuperado de http:// pepsic.bvsalud.org/scielo.php?script=sci_arttext\& pid $=$ S1413-85572002000100002

Houaiss, A., \& Villar, M. (2012). Grande dicionário Houaiss Beta da lingua portuguesa. Versão eletrônica. Recuperado de http://houaiss. uol. com. br.

Houaiss, A., Villar, M., \& de Mello Franco, F. M. (2001). Minidicionário Houaiss da língua portuguesa. Objetiva.

Marini, J. A. S. (2012). Aprendizagem autorregulada de estudantes de Pedagogia: Suas estratégias de aprendizagem, teorias implícitas de inteligência e variáveis motivacionais (Tese de doutorado). Faculdade de Educação da Universidade Estadual de Campinas. Recuperado de http://repositorio.unicamp.br/jspui/handle/ REPOSIP/250749

Neves, E. R. C., \& Boruchovitch, E. (2007). Escala de avaliação da motivação para aprender de alunos do ensino fundamental (EMA). Psicologia: Reflexão e Crítica, 20(3), 406-413. Recuperado de http:// www.scielo.br/pdf/prc/v20n3/a08v20n3

Rodrigues, M. S. P., \& Leitão, G. D. C. M. (2000). Estágio curricular supervisionado com ênfase no desenvolvimento da autonomia e da responsabilidade. Texto \& Contexto Enfermagem, 9(3), 216-229. Recuperado de http://bases.bireme.br/cgi-bin/ wxislind.exe/iah/online/?IsisScript $=$ iah/iah.xis\& src $=$ google $\&$ base $=$ BDENF\&lang $=$ p\&nextAction $=\operatorname{lnk} \&$ exprSearch $=193 \&$ indexSearch $=$ ID

Ruiz, V. M., \& Witter, G. P. (2005). Produção científica sobre motivação do universitário na base ERIC (1992-2002). Em G. P. Winter (Org.). Metaciência e Psicologia (pp. 93-110). Campinas: Alínea.

Ruthes, R. M., Feldman, L. B., \& Olm Cunha, I. C. K. (2010). Foco no cliente: Ferramenta essencial na gestão por competência em enfermagem. Revista Brasileira de Enfermagem, 63(2). Recuperado de http://clacso.m.redalyc.org/articulo.oa?id $=267019594023$
Santos, A. A. A., Mognon, J. F., Alcará, A. R., \& Lemos, T. H. (2011). Motivação para aprender: Evidência de validade convergente entre duas medidas. Aletheia, (35-36), 36-50. Recuperado de http://clacso.m.redalyc.org/articulo. oa?id=115025560004

Santos, A. A. A., Polydoro, S. A. J., Teixeira, M. A., \& Bardagi, M. P. (2010). Avaliação da integração do aluno ao ensino superior brasileiro. Em A. A. A. Santos, F. F. Sisto, E. Boruchovitch \& E. Nascimento (Orgs.), Perspectivas em Avaliação Psicológica (pp.165-188). São Paulo: Casa do Psicólogo.

Soares, A. B., Francischetto, V., Dutra, B. M., Miranda, J. M., Nogueira, C. C. C., Leme, V. R., Araújo, A. M., \& Almeida, L. S. (2014). O impacto das expectativas na adaptação acadêmica dos estudantes no Ensino Superior. Psico-USF, 19(1), 49-60. Recuperado de http://hdl.handle.net/1822/29263

Sobral, D. T. (2003). Motivação do aprendiz de medicina: Uso da escala de motivação acadêmica. Psicologia: Teoria e Pesquisa, 19(1), 25-31. Recuperado de http://www.scielo.br/pdf/\%0D/ptp/v19n1/ a05v19n1.pdf

Tabachnick, B. G., \& Fidell, L. S. (2007). Using multivariate statistics. Boston, MA: Allyn \& Bacon/Pearson Education.

Wehmeyer, M. L. (1999). A functional model of self-determination: Describing development and implementing instruction. Focus on Autism and other Developmental Disabilities, 14(1), 53-61. doi:10.1177/108835769901400107

Zenorini, R. P. C., \& Santos, A. A. A. (2003). A motivação e a utilização de estratégias de aprendizagem em universitários. Em E. Mercuri \& S. A. J. Polydoro (Orgs.). Estudante Universitário: Caracteristica e Experiências de Formação (pp. 67-86). Taubaté, SP: Cabral.

Recebido em: 29/03/2018

Reformulado em: 05/09/2018 
Sobre os autores:

Leandro Dias de Araújo é fisioterapeuta, doutor em Psicologia pela Universidade Salgado de Oliveira, mestre em Ensino na Saúde na Universidade Gama Filho, especialista em Psicomotricidade pela Universidade Estácio de Sá e professor titular dos cursos de graduação e pós-graduação em Fisioterapia, Educação Física e Psicomotricidade das Universidades Salgado de Oliveira e Centro Universitário Celso Lisboa.

ORCID: https://orcid.org/0000-0002-3898-1153

E-mail: leandiar@hotmail.com

Márcia Maria Peruzzi Elia da Mota é psicóloga, doutora em Psicologia pela University of Oxford (1996), mestre em Métodos de Pesquisa em Psicologia na Universidade de Reading, também na Inglaterra, diretora do Instituto de Psicologia da UERJ, coordenadora do grupo de pesquisa do CNPq Cognição e Contextos Sociais e professora titular do Programa de Pós-Graduação em Psicologia da Universidade Salgado de Oliveira.

ORCID: https://orcid.org/0000-0002-8343-0641

E-mail:mmotapsi@gmail.com

Contato com os autores:

Rua Marechal Deodoro, 263, Centro

Niterói-RJ, Brasil

CEP: 24030-060 